\title{
Role of the route of leukotrienes in an experimental model of oral mucositis induced by 5 -fluorouracil ${ }^{1}$
}

\author{
Viviane Carvalho da Silva', Renata Ferreira de Carvalho Leitão", Gerly Anne de Castro Brito", Conceição \\ da Silva Martins", Gildenio Estevam Freire"II, Karoline Saboia Aragão'v, Carlos Wagner de Souza Wanderley , \\ Marcos Rabelo de Freitas ${ }^{\mathrm{VI}}$
}

\begin{abstract}
'Fellow PhD degree, Postgraduate Program in Medical and Surgical Sciences, Department of Surgery, School of Medicine, Universidade Federal do Ceará (UFCE), Fortaleza-CE, Brazil. Conception and design of the study, acquisition and interpretation of data, statistics analysis, manuscript preparation, final approval.

"PhD, Postgraduate Program in Morphofunctional Sciences, Department of Morphology, School of Medicine, UFCE, Fortaleza-CE, Brazil. Acquisition and interpretation of data, histopathological examinations, manuscript preparation.

'"Fellow Master degree, Postgraduate Program in Morphofunctional Sciences, Department of Morphology, School of Medicine, UFCE, Fortaleza-CE, Brazil. Acquisition and interpretation of data, manuscript preparation.

IVPhD, Laboratory of Inflammation and Cancer Pharmacology (LAFICA), UFCE, Fortaleza-CE, Brazil. Conception and design of the study, acquisition and interpretation of data, manuscript preparation.

${ }^{\vee}$ Fellow PhD degree, LAFICA, UFCE, Fortaleza-CE, Brazil. Acquisition and interpretation of data, manuscript preparation. V'PhD, Postgraduate Program in Medical and Surgical Sciences, Department of Surgery, Faculty of Medicine, UFCE, Fortaleza-CE, Brazil. Conception and design of the study, interpretation of data, manuscript preparation, final approval.
\end{abstract}

\section{Abstract}

Purpose: To investigate the participation of cysteinyl leukotrienes in the pathophysiology of oral mucositis.

Methods: Oral mucositis was induced in hamsters using 5-fluorouracil (5-FU; 60 and $40 \mathrm{mg}$ / kg; i.p., on days 1 and 2, respectively, and with excoriations in jugal mucosa on day 4). Montelukast (10, 20, or $40 \mathrm{mg} / \mathrm{kg} / \mathrm{d}$; gavage), MK886 (3 mg/kg/d, i.p.), or saline or celecoxib (7.5 $\mathrm{mg} / \mathrm{kg} / \mathrm{d}$; i.p.) was administered $1 \mathrm{~h}$ prior to 5-FU and daily, until the fourth (MK886) or tenth day, when the animals were euthanized and their jugal mucosa was collected for macroscopic, histopathological, and immunohistochemical evaluation.

Results: Neither montelukast nor MK-886 prevented the oral mucositis induced by 5-FU, as observed by histopathological evaluation. In addition, we did not find significant differences in the expression of inducible nitric oxide synthase-2, cyclooxygenase-2, or interleukin (IL)-1 $\beta$ between the experimental and control groups. However, we did observe a significant decrease in tumor necrosis factor (TNF)- $\alpha$ expression for all doses of montelukast; we also observed a significant decrease in IL-10 with 40 mg/kg/d and MK 886.

Conclusions: Cysteinyl leukotrienes do not play an important role in experimental oral mucositis induced by 5-FU. There is a modulating action specifically on TNF- $\alpha$.

Key words: Leukotrienes. Arachidonate 5-Lipoxygenase. Stomatitis. Fluorouracil. Cricetinae. 


\section{- Introduction}

Oral mucositis (OM) is an important, dose-limiting side effect observed in patients undergoing radiotherapy (RT) and chemotherapy $\quad(\mathrm{CT})^{1,2}$. Proinflammatory cytokines are released in response to reactive oxygen species produced by CT-damaged cells; the pathogenesis of OM, however, is yet to be fully elucidated ${ }^{3}$.

\section{Cyclooxygenase (COX)-2 and} 5-lipoxygenase (5-LOX) are key enzymes involved in the metabolism of arachidonic acid (AA); these enzymes lead to the formation of fatty acids known as eicosanoids ${ }^{4}$. The COX pathway helps mediate the inflammatory response; many chemotherapeutic drugs can directly activate this pathway at several sites, including the oral mucosa4.

Despite the well-known relationship of the COX pathway with chemotherapy-induced $\mathrm{OM}^{4,5}$, we could not find any study investigating the role of leukotrienes (AA metabolites) in OM caused by antineoplastic agents. It has been proposed that the inhibition of one arm of the AA cascade may result in amplification of another arm, with potentially undesirable effects; for example, inhibition of the COX-2 pathway may lead to activation of 5-LOX and increased production of leukotrienes such as LTD46.

Studies have identified leukotrienes as mediators in the inflammatory processes of the gastric and intestinal mucosa $a^{7-10}$. It is known that oral and gastrointestinal mucositis present similarities in their pathophysiology. Therefore, elucidating the pathophysiological mechanisms by which leukotrienes participate in inflammatory processes of the oral mucosa may lead to methods of prevention and control. The present study was performed to investigate the participation of cysteinyl leukotrienes (cysLT) in 5-FU induced OM pathophysiology, using montelukast (MTK), (blocker of the cysteinyl leukotrienes receptor) and MK-886 (5-LOX inhibitor), while evaluating inflammatory parameters such as cytokines.

\section{Methods}

All procedures involving animals were approved by Universidade Federal do Ceará, Committee on ethical treatment of research animals (protocol 0033/2012). Surgical procedures and animal treatments were conducted in accordance with the Guidelines for Institutional Animal Care, and were approved by our local ethics committee for experimental use on the animals.

Eighty-four adult male golden hamsters weighing $100-150$ g were housed in temperature-controlled rooms and received water and food ad libitum.

\section{Drugs}

5-FU $\left(25 \mathrm{mg} / \mathrm{mL}\right.$; Fluoro-acil ${ }^{\circ}$, Roche, Rio de Janeiro, Brazil); MTK (Singulair ${ }^{\circ}$, Merck Sharp and Dohme, Hoddesdon, HRT, United Kingdom); MK 886 (MK-886 sodium salt hydrate- (M2692 SIGMA) $>98 \%$ (HPLC) -Synonym: 3-[3-tert-Butylthio-1-(4chlorobenzyl)-5-isopropyl-1H-indol-2-yl]-2,2dimethylpropionic acid, sodium salt hydrate); celecoxib (Celebra', capsules com $200 \mathrm{mg}$, Pfizer, São Paulo, Brazil); tribromoethanol 2,5\% (25 mg/mL).

\section{Induction of experimental OM}

$\mathrm{OM}$ was induced using two intraperitoneal (i.p.) administrations of 5-FU on days 1 and 2 of the experiment (60 and $40 \mathrm{mg} /$ $\mathrm{kg}$, respectively), according to an experimental OM model previously described ${ }^{11,12}$. On day 4 , under anesthesia with $2.5 \%$ tribromoethanol $(250 \mathrm{mg} / \mathrm{kg}$, i.p.), the cheek pouch mucosa was irritated by superficial scratching, to potentiate $\mathrm{OM}$. We performed this step by dragging the 
tip of an 18-gauge needle twice, in a linear manner, across the everted cheek pouch. The animals were killed on day 10 after the initial injection of 5-FU, under anesthesia with $2.5 \%$ tribromoethanol $(250 \mathrm{mg} / \mathrm{kg}$, i.p.). There were at least 5 animals in each experimental group.

\section{Experimental groups}

- MTK10: animals subjected to OM induction that received MTK $(10 \mathrm{mg} / \mathrm{kg} / \mathrm{d}$; via gavage) $1 \mathrm{~h}$ before the first administration of 5-FU, and daily for $10 \mathrm{~d}$.

- MTK20: animals subjected to OM induction receiving MTK $(20 \mathrm{mg} / \mathrm{kg} / \mathrm{d}$; via gavage) $1 \mathrm{~h}$ before the first administration of 5-FU, and daily for $10 \mathrm{~d}$.

- MTK40: animals subjected to OM induction receiving MTK $(40 \mathrm{mg} / \mathrm{kg} / \mathrm{d}$; via gavage) $1 \mathrm{~h}$ before the first administration of 5-FU, and daily for $10 \mathrm{~d}$.

- MK886: animals subjected to OM induction that received MK886 (3 mg/kg/d, i.p.) $1 \mathrm{~h}$ prior to the first administration of 5-FU, and daily for $4 \mathrm{~d}$.

The control groups were constituted of at least 5 animals per group, and were organized as follows:

- 5-FU: animals subjected to OM induction

- SAL: animals not subjected to OM induction, that is, normal control.

- MT: animals subjected only to excoriations in the oral mucosa

- CLX: animals subjected to OM induction receiving celecoxib $(7.5 \mathrm{mg} / \mathrm{kg} / \mathrm{d}$; i.p.) $1 \mathrm{~h}$ before the first administration of 5-FU, and daily for $10 \mathrm{~d}$. This group was added as a gold standard treatment, in light of previous findings that this intervention considerably reduced the inflammatory effects of induced OM.

The study was divided into two parts: the first set of experiments investigated the effect of blockade of cys-LT via MTK $(10,20$, or $40 \mathrm{mg} / \mathrm{kg} / \mathrm{d}$, gavage) for $10 \mathrm{~d}$. The second set of experiments investigated the cascade blockade of leukotriene synthesis at a step further upstream, thus inhibiting all classes of leukotrienes, and not only the cys-LT, for which inhibition was assessed after the administration of MTK. MK886 (3 mg/kg/d, i.p.) was therefore used as an inhibitor of 5-LOX synthesis, $1 \mathrm{~h}$ prior to 5-FU administration, and daily until experimental day 4. The use of this drug for $4 \mathrm{~d}$ was based on previous studies, using the same experimental model as that used in the present study, suggesting that an intervention in the first $4 \mathrm{~d}$ is enough to prevent or minimize the inflammatory changes observed on day $10^{13}$. The control groups used were the same as described for the first set of experiments, except for the celecoxib group (CLX). Likewise, the animals were euthanized on day 10 of the second experiment set.

\section{Macroscopic analysis of cheek pouch}

Photographs were used for scoring lesions. For macroscopic analysis, inflammatory aspects such as erythema, erosion, vasodilatation, epithelial ulcerations, and abscesses were evaluated in a singleblind fashion, and graded as follows. Score 0: completely healthy cheek pouch, with erosion or vasodilatation absent. Score 1: presence of erythema, but no evidence of erosion in the cheek pouch. Score 2: severe erythema, vasodilation, and surface erosion. Score 3: formation of ulcers on one or more faces of the mucosa, but not affecting more than $25 \%$ of the surface area of the cheek pouch; severe erythema and vasodilatation. Score 4: Cumulative formation of ulcers on about $50 \%$ of the surface area of the cheek pouch. Score 5: virtually complete ulceration of the cheek pouch mucosa. In the latter case, the fibrosis makes oral mucosa exposure difficult. 


\section{Histopathological analysis of cheek pouch}

The specimens were fixed in $10 \%$ neutral buffered formalin, dehydrated, and embedded in paraffin. Sections of 4- $\mu \mathrm{m}$ thickness were obtained for hematoxylin-eosin staining (H\&E), and examined using light microscopy (x 40). The parameters of inflammatory cell infiltration, vasodilatation, presence of hemorrhagic areas, edema, ulcerations, and abscesses were determined in a single-blind fashion and graded as follows. Score 0: normal epithelium and connective tissue without vasodilatation; absence of discreet cellular infiltration; absence of hemorrhagic areas, ulcerations, or abscesses. Score 1: discreet vasodilatation, re-epithelization areas; discreet inflammatory infiltration with mononuclear prevalence; absence of hemorrhagic areas, edema, ulcerations, or abscesses. Score 2: moderate vasodilatation, areas of hydropic epithelial degeneration, inflammatory infiltration with neutrophil prevalence, presence of hemorrhagic areas, edema and eventual ulcerations, and absence of abscesses. Score 3: severe vasodilatation, inflammatory infiltration with neutrophil prevalence, presence of hemorrhagic areas, edema and extensive ulceration, and abscesses.

Immunohistochemical detection of iNOS2, COX-2, IL1- 6, TNF- $\alpha$, and IL1O

On day 10 of the experiment, the animals were euthanized and had their oral mucosa removed and fixed in $10 \%$ formalin for $24 \mathrm{~h}$, in the preparation for immunohistochemistry slides. Immunohistochemistry for iNOS2, COX-2, IL1- $\beta$, TNF- $\alpha$, and IL10 was performed using the streptavidin-biotin-peroxidase method $^{14}$. The tissues were dehydrated in alcohol, and subsequently embedded in paraffin. After this procedure, $4-\mu \mathrm{m}$ serial cuts were made in appropriate microtome and placed on L-polylysine slides suitable for immunohistochemistry. The sections were dewaxed, hydrated in xylol and alcohol, and for antigen recovery, immersed in $0.1 \mathrm{M}$ citrate buffer ( $\mathrm{pH} 6.0$ or 9.0) in a warm water bath for 30 $\min$ at $95^{\circ} \mathrm{C}$. After cooling to room temperature for $20 \mathrm{~min}$, the sections were washed with phosphate buffered saline (PBS), interspersed with blocking the endogenous peroxidase with $3 \% \mathrm{H}_{2} \mathrm{O}_{2}$ solution $(20 \mathrm{~min}$ ). Protein in block was next blocked for $20 \mathrm{~min}$. The sections were incubated overnight with goat antiiNOS2 (1: 200), COX-2 (1: 100), IL1- $\beta$ (1:00), TNF- $\alpha$ (1: 100), and IL10 (1: 100). All antibodies (Santa Cruz) were diluted in antibody diluents (Abican). After washing in PBS, incubation with bituminized (goat) IgG anti-goat, rabbit, and anti-mouse diluted 1: 400 (Santa Cruz) was performed for $30 \mathrm{~min}$. After washing, the sections were incubated with the conjugated streptavidin peroxidase complex (ABC Santa Cruz complex) for $30 \mathrm{~min}$. After further washing with PBS, staining with the chromogen 3,3'-diaminobenzidine peroxide (DAB), and counterstaining with Mayer's hematoxylin, the samples were finally dehydrated, and the slides were assembled. Negative controls were processed simultaneously as described above, with the primary antibody being replaced with $5 \%$ PBS-BSA.

Quantification of the immunostained cells was performed by using ImageJ to process images captured using a light microscope coupled to a camera with an LAZ 3,5 acquisition system (Leica DM1000, Germany). At least 4 animals per group were evaluated, considering 10 fields by histological section, at x400 magnification. Immunostained cells were those that showed brown cytoplasmic staining. The results were expressed as the number of cells marked per group.

\section{Statistical analysis}

Data were expressed as mean \pm standard error (mean \pm SEM), or median, as appropriate. Before choosing the statistical test 
(parametric or non-parametric), the groups compared were assessed for their normality using the D'Agostino \& Pearson omnibus and Shapiro-Wilk tests. Analysis of variance (ANOVA) using the Kruskal-Wallis test, followed by Dunn's multiple comparisons test, was used to compare data from the various groups. The data were analyzed using the GraphPad Prism 6.0 software package (La Jolla, CA, USA). The level of significance was $5 \%(p<0.05)$.

\section{Results}

\section{First set of experiments}

Macroscopic and histopathological analyses of cheek pouch

Atday 10 of the experiment, macroscopic analysis showed that the control group subjected to OM induction by 5-FU presented significant inflammatory alterations, relative to controls not subjected to OM induction (SAL and MT) $(p<0.05)$. Histopathological analysis (H\&E) corroborates these findings. This group had a median histopathological score of 3 , and a significant variation in relation to the groups SAL, TM, and CLX (Table 1) $(p<0.05)$.

MTK at concentrations of 10 and 20 $\mathrm{mg} / \mathrm{kg} / \mathrm{d}$ did not prevent macroscopic damage to the oral mucosa, but animals treated with the highest dose of MTK presented lower scores than those in the 5-FU group. The MTK40 group did not have a statistically significant difference from the $S A L, M T$, or CLX groups (normal control and standard treatment groups). However, no statistical differences were found between the MTK40 and 5-FU groups. A partial protective effect was suggested for $40 \mathrm{mg} / \mathrm{kg}$ of MTK. However, this protective effect was not confirmed in the histopathological analysis (Table 1$)(p<0.05)$. Celecoxib significantly prevented oral mucosal damage when compared to the 5-FU group, as expected (Table 1$)(p<0.05)$.

Table 1 - Macroscopic and microscopic evaluation of oral mucositis of animals with $10 \mathrm{~d}$ of treatment.

\begin{tabular}{llllllll}
\hline & & & \multicolumn{5}{c}{ 5-FU } \\
Experimental groups & SAL & TM & 5-FU & CLX & MTK10 & MTK20 & MTK40 \\
\hline Macroscopic analysis & $0(0-0)$ & $1.5(0-3) \#$ & $4.0(3-5)^{*}$ & $2.0(0-4) \#$ & $3.5(3-4)^{*}$ & $4.0(3-5)^{*}+$ & $3.0(1-3)$ \\
Histopathological analysis & $0(0-2)$ & $1.0(0-3) \#$ & $3.0(2-3)^{*}$ & $1.0(0-1) \#$ & $3.0(3-3)^{*}+\$$ & $3.0(1-3)^{*}+$ & $3.0(1-3)^{*}$ \\
\hline
\end{tabular}

The data represent medians of the scores (and variation) of at least five animals per group. ${ }^{*} \mathrm{P}<0.05$ represents statistical difference in relation to the control group constituted of healthy animals $(S A L) ;+P<0.05$ represents statistical difference in relation to the control group composed of animals subjected to mechanical trauma (MT); $\$ \mathrm{P}<0.05$ represents statistical difference in relation to the $\mathrm{CLX}$ control group; \# P<0.05 compared to the 5-FU group. Kruskal-Wallis and Dunn's test.

Immunohistochemical detection of iNOS2, COX-2, IL1- $\beta$, TNF- $\alpha$, and IL10

The immunohistochemical analysis for iNOS2 allows us to infer the production of nitric oxide (NO) in the mucosa. Therefore, it is a parameter of inflammatory intensity. It was observed that 5-FU-induced mucositis resulted in a significant increase in the expression of iNOS2 in the jugal mucosa of hamsters (Figure 1) $(p<0.05)$; these data demonstrate that NO participation through iNOS2 increased due to induction of mucositis. Treatment with MTK did not prevent the increase in iNOS2 expression (Figure 1) $(p<0.05)$. The CLX group presented a significant reduction in the cell's immunomarcation, demonstrating a protective action. Thus, it can be seen that cys-LTs in this study exerted little influence on the increase in the number of cells marked for iNOS2 and, consequently, little influence on NO production. 

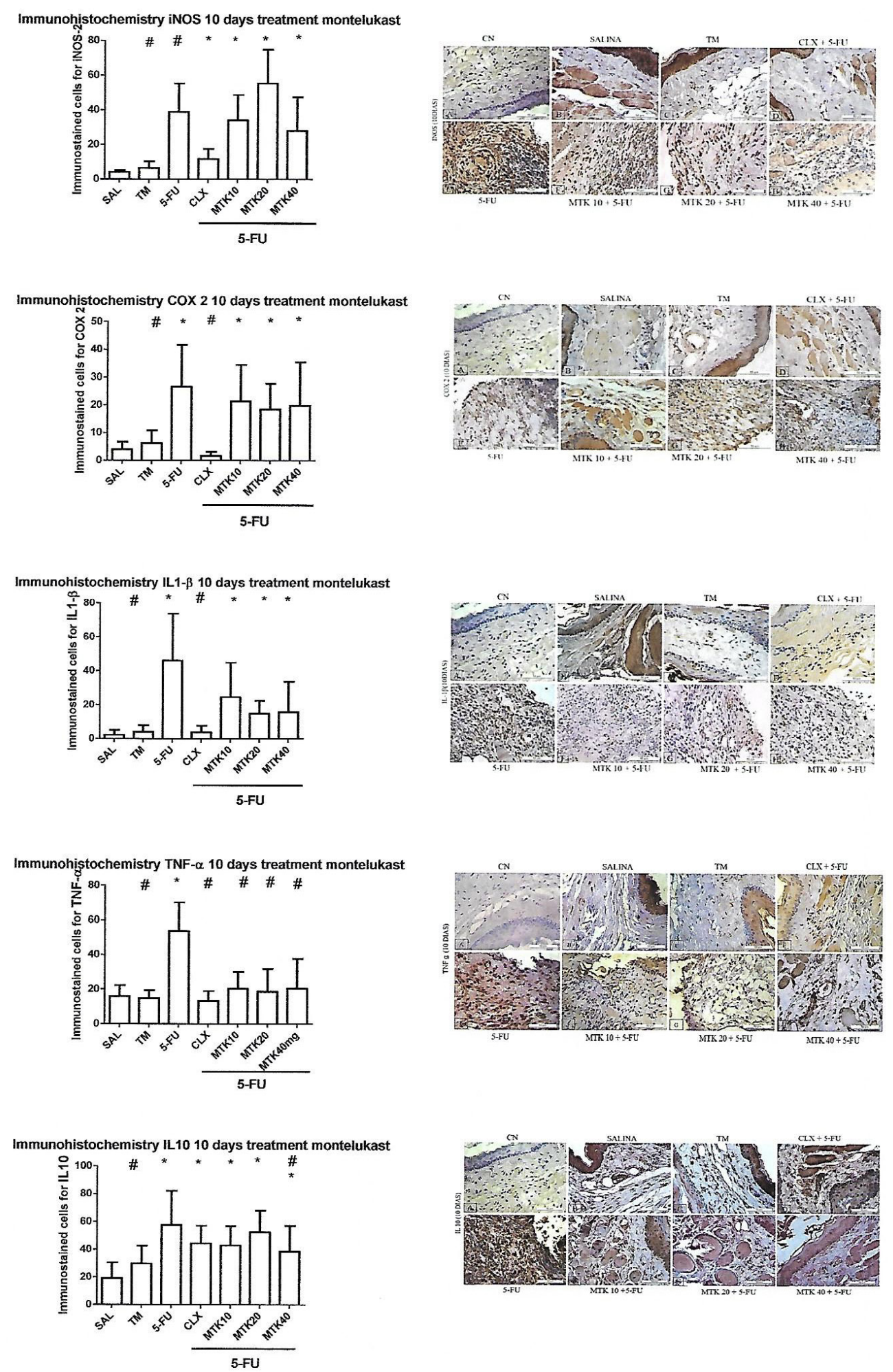

Figure 1 - Immunohistochemical plot and photomicrographs representative of immunohistochemical detection of iNOS2, COX2, IL1- $\beta$, TNF- $\alpha$, and IL10 with $10 \mathrm{~d}$ treatment of montelukast. 
On day 10 of the experiment, the animals were sacrificed and the jugal mucosa samples were processed for immunohistochemistry of iNOS2, COX2, IL1- $\beta$, TNF- $\alpha$, and IL10 (x400 magnification). (A) Negative control (CN) represents a sample of jugal mucosa where the primary antibody was replaced with $5 \%$ PBS-BSA and shows no labeling; (B) normal hamster jugal mucosa (without induction of oral mucositis and receiving saline (SAL); (C) jugal mucosa of hamsters submitted only to mechanical trauma (TM); (D) jugal mucosa of hamsters submitted to 5-FU induced oral mucositis who received celecoxib $7.5 \mathrm{mg} / \mathrm{d}$ for 10 d, i.p.); (E) oral mucosa of animals submitted to 5-FU-induced oral mucositis receiving saline (5-FU); (F) oral mucosa of animals submitted to 5-FU induced oral mucositis receiving montelukast $10 \mathrm{mg} / \mathrm{kg} / \mathrm{d}$ for $10 \mathrm{~d}$ (MTK10); (G) oral mucosa of animals submitted to oral mucositis induced by 5 -FU receiving montelukast $20 \mathrm{mg} / \mathrm{kg} / \mathrm{d}$ for $10 \mathrm{~d}$ (MTK20); (H) oral mucosa of animals submitted to mucositis induced by 5 -FU that received montelukast 40 $\mathrm{mg} / \mathrm{kg} / \mathrm{d}$ for $10 \mathrm{~d}$ (MTK40). The data represent medians of the scores (and variation) of at least five animals per group. ${ }^{*} \mathrm{P}<0.05$ represents statistical difference in relation to the control group constituted of healthy animals (SAL); $\# p<0.05$ compared to the 5-FU group. KruskalWallis and Dunn's test. At least 4 animals per group were evaluated, 10 fields were analyzed by histological section at $\times 400$ magnification, and the counting of positive cells marked by each field was performed using ImageJ. Cells considered positive are marked with cytoplasmic brown staining.

In order to analyze the blockade of the cys-LTs pathway in comparison to that of the COX-2 pathway, immunostaining of COX-2 was quantitatively assessed. 5-FUinduced mucositis in the jugal mucosa of the animals promoted a significant increase in the expression of COX-2-labeled cells, supporting the strong stimulation of the cyclooxygenase pathway by this inflammatory stimulus. However, in the CLX group, as expected, there was a significant reduction in the number of immunostained cells (Figure 1$)(p<0.05)$. Cys-LTs do not significantly interfere in the mediation of COX-2 expression and its blockade therefore cannot exert a protective effect on 5-FU-induced OM. The findings are similar to the immunohistochemistry results for iNOS2 (Figure 1) $(p<0.05)$. This similarity corroborates an apparent interaction between the COX2 pathway and the production of iNOS2; however, it also suggests that the same does not occur in relation to cys-LTs and iNOS2 in OM caused by 5-FU. In addition, the use of the cys-LT blocker apparently had no influence on COX-2 expression and inflammation mediated by this pathway (Figure 1$)(p<0.05)$.

IL1- $\beta$ is a cytokine with recognized proinflammatory activity in 5-FU-induced OM. The 5-FU group presented a significant increase in the immunostaining for IL1- $\beta$ relative to the healthy controls (SAL and MT) (Figure 1) $(p<0.05)$, as well as the control considered to be the gold standard of treatment by this study (CLX). There were no significant differences in the groups that used MTK at any of the doses administered, although a decreasing tendency was observed, which was not confirmed by the statistical tests (Figure 1$)(p<0.05)$. The presence of IL1- $\beta$-labeled cells followed the same pattern as that observed for INOS2 and COX-2 (Figure 1) $(p<0.05)$.

We performed immunohistochemical investigation of TNF- $\alpha$, another important proinflammatory cytokine, and found a significant increase of its expression in the 5 -FU group (Figure 1$)(p<0.05)$. Consistent with inflammatory activity of TNF- $\alpha$ in this group, the administration of celecoxib promoted a significant reduction in the expression of this cytokine, as well as the inhibition of cysLT through the use of MTK at various doses (Figure 1) $(p<0.05)$, demonstrating a protection or decrease in this inflammatory marker by inhibition of both the cyclooxygenase-2 pathway and the cys-leukotriene pathway. These findings suggest the possibility of cys-LT having an influence on the production of TNF- $\alpha$ 
in OM caused by 5-FU, and consequently a partial protective or modulatory effect on this inflammatory parameter.

To verify the behavior of an antiinflammatory cytokine to the effect of the inhibition of cys-LT and COX-2 during OM induced by 5-FU, an immunohistochemical study for the detection of IL10 was performed. All 5-FU-induced OM groups (5-FU, MTK10, MTK20, MTK40, and CLX) exhibited an increase in IL10-labeled cells on jugal mucosa of the hamsters (Figure 1$)(p<0.05)$. The CLX group (standard treatment) also showed a significant increase in expression when compared with normal controls (not subjected to OM induction) (Figure 1) $(p<0.05)$. When 5-FU was the target group for comparative analysis, it was statistically different from saline groups, mechanical trauma and, interestingly, from the group administered MTK at a dose of $40 \mathrm{mg} /$ $\mathrm{kg} / \mathrm{d}$ (Figure 1) $(\mathrm{p}<0.05)$. However, the 5-FU group did not present a significant difference from the groups that were administered 10 and $20 \mathrm{mg} / \mathrm{kg}$ doses per day of MTK and the group that received celecoxib (Figure 1$)(p<0.05)$. These findings suggest an influence of cysLT on IL10 expression behavior for less, with increased blockade of these. However, they also demonstrate increased IL10 expression despite COX-2 blockade in the 5-FU-induced OM model.

Second set of experiments: evaluation after 5-lipoxygenase blockade by MK886

Blocking of 5-lipoxygenase by MK886 inhibits the leukotriene pathway at its root, thus preventing the production of both cys-LT and other leukotrienes. This blocking method can therefore reveal whether the other leukotrienes, besides the cys-LT, have different roles in OM induced by 5-FU.

Macroscopic and Histopathological analysis of cheek pouch

The macroscopic evaluation of lesions occurring on the mucosa of the hamster with
5-FU induced OM showed, in the groups where there was induction of $\mathrm{OM}$, alterations suggestive of intense inflammatory processes (Table 2) $(p<0.05)$. Administration of the 5-LOX blocker (MK886) was not able to decrease the intensity of the inflammatory signals. Histopathological evaluation showed similar findings, corroborating that the blockade of all leukotriene classes was not able to decrease the intensity of OM (Table 2$)(p<0.05)$. These data demonstrate that leukotrienes do not play an important role in mediating this inflammatory process.

Table 2 - Macroscopic and microscopic evaluation of oral mucositis of the animals with $4 \mathrm{~d}$ of treatment.

\begin{tabular}{lcllc}
\hline $\begin{array}{l}\text { Experimental } \\
\text { groups }\end{array}$ & SAL & TM & 5-FU & $\begin{array}{c}\text { MK886 } \\
+5-F U\end{array}$ \\
\hline $\begin{array}{l}\text { Macroscopic } \\
\text { analysis }\end{array}$ & 0 & 2 & 4 & 3 \\
Histopathological & 0 & $1(0-$ & 3 & 3 \\
analysis & $(0-2)$ & $3) \#$ & $(2-3)^{*}+$ & $(3-3)^{*+}$ \\
\hline
\end{tabular}

The data represent medians of the scores (and variation) of at least five animals per group. ${ }^{*} \mathrm{P}<0.05$ represents statistical difference in relation to the control group constituted of healthy animals (SAL); $+\mathrm{P}<0.05$ represents statistical difference in relation to the control group composed of animals subjected to mechanical trauma (MT); \#P<0.05 compared to the $5-\mathrm{FU}$ group. Kruskal-Wallis and Dunn's test.

Immunohistochemistry for detection of iNOS2, COX-2, IL1- $\beta$, TNF- $\alpha$, and IL10

To evaluate the action of 5-LOX block on the production of nitric oxide (NO) in OM induced by 5-FU, we performed the quantitative analysis of the cellular marking by immunohistochemistry for iNOS2. 5-FUinduced mucositis resulted in a significant increase in iNOS2 in the jugal mucosa of hamsters (Figure 2) $(p<0.05)$. Treatment with MK886 was not able to prevent the increase of iNOS2 expression (Figure 2$)(p<0.05)$. This result indicates that leukotrienes have no significant role in iNOS2 expression, as their blockade was unable to ameliorate mucositis in the OM model of hamsters induced by 5-FU. 

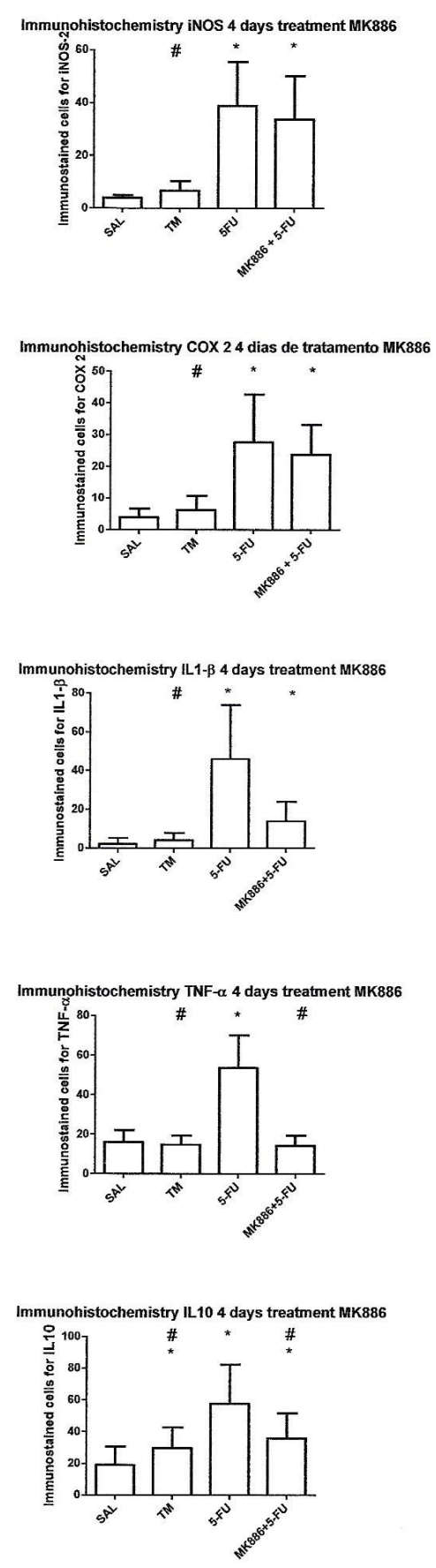
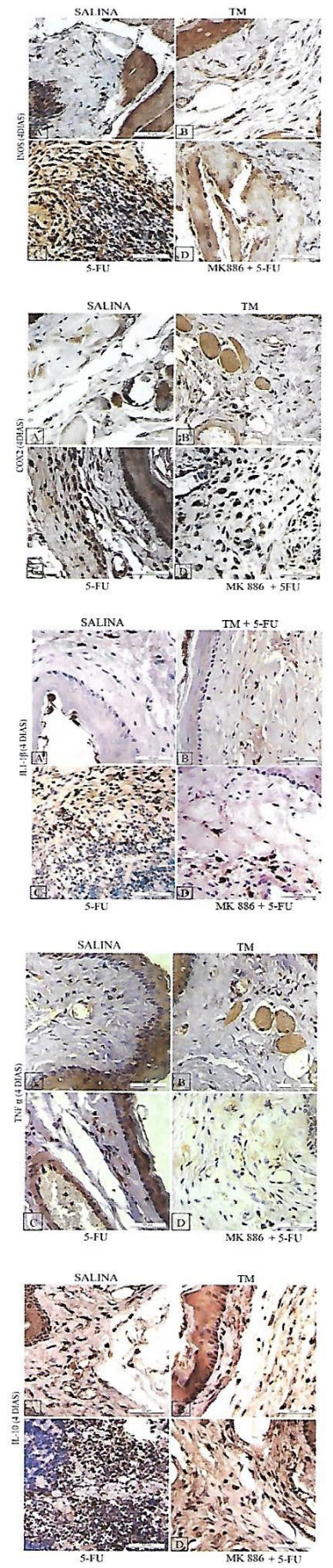

Figure 2 - Immunohistochemical plot and photomicrographs representative of immunohistochemistry for detection of iNOS2, COX2, IL1- $\beta$, TNF- $\alpha$, and IL10 with $4 \mathrm{~d}$ treatment of MK886. On day 10 of the experiment, the animals were sacrificed and the jugal mucosa samples were processed to detect iNOS2, COX2, IL1- $\beta$, TNF- $\alpha$, and IL10 using immunohistochemistry (x400x magnification). (A) Jugal mucosa of normal hamsters, no induction of oral mucositis, saline (SAL); (B) Hamster jugal mucosa submitted only to mechanical trauma (TM); (C) Oral mucosa of animals submitted to oral mucositis induced by 5-FU that received saline (5-FU); (D) Oral mucosa of hamsters submitted to 5-FU induced oral mucositis receiving MK886 $3.0 \mathrm{mg} / \mathrm{d}$ for 4d, i.p. The data represent medians of the scores (and variation) of at least five animals per group. ${ }^{*} \mathrm{P}<0.05$ represents statistical difference in relation to the control group. 
In order to analyze the importance of the COX-2 pathway after 5-LOX blockade, the immunostaining of COX-2 cells was evaluated quantitatively. The induction of 5-FU mucositis in the jugal mucosa of the animals promoted a significant increase in the number of COX-2labeled cells, confirming the stimulation of the cyclooxygenase pathway by this inflammatory stimulus (Figure 2$)(p<0.05)$. The administration of MK886 to animals that underwent OM induction, therefore, was not able to promote a significant reduction in the number of marked cells when the groups were compared with the 5 -FU control (Figure 2) $(p<0.05)$. Thus, even a blockade of all leukotriene classes did not interfere with COX-2 expression in OM induced by 5 -FU.

IL1- $\beta$ expression was carried out to detect whether the production of this proinflammatory cytokine was modified due to the inhibition of 5-LOX by MK886 in OM induced by 5 -FU. Increased number of IL1- $\beta$ immunolabeled cells was observed in the OM groups. There was no significant difference between the MK 886 group and the 5 -FU group (Figure 2) $(p<0.05)$. Although the group in which 5-LOX was inhibited showed a decreasing tendency for IL1- $\beta$-immunolabeled cells, this tendency was not confirmed by statistical tests (Figure 2) $(p<0.05)$. We thus conclude that blocking all classes of leukotrienes does not significantly interfere in the expression of this important inflammatory cytokine for 5-FUinduced OM.

Immunohistochemical observations revealed a significant increase in TNF- $\alpha$ expression in the 5-FU group (positive control of OM induction), thus, demonstrating their inflammatory action in this group (Figure 2) $(p<0.05)$. Administration of MK886 promoted a significant reduction in the number of cells labeled for this cytokine. Thus, protection was demonstrated by the decrease of this inflammatory marker, by inhibition of the leukotriene pathway. These findings reinforce that leukotrienes influence the production of TNF- $\alpha$ in OM induced by 5-FU, and consequently exert a partially protective or modulatory effect on this important inflammatory parameter.

The immunohistochemical detection of IL10 was performed to verify the behavior of a cytokine with anti-inflammatory characteristics as an effect of 5-LOX inhibition during $\mathrm{OM}$ induced by 5-FU. All groups with induction of $\mathrm{OM}$ by 5-FU (5-FU, MK886) showed an increase in the expression of IL10 on jugal mucosa cells when compared to the SAL group (Figure 2) $(p<0.05)$. Interestingly, the MT group also showed a significant increase in expression when compared to SAL groups (both normal controls, not subjected to OM induction) (Figure 2) $(p<0.05)$. The analysis of cells labeled for IL10 shows a statistically significant difference between the saline group and all others, including the MT group (Figure 2) $(p<0.05)$. On the other hand, a significant difference was observed between the 5-FU group and the saline, MT, and MK886 groups. That is, inhibition of leukotrienes by blocking 5-LOX for only $4 \mathrm{~d}$ interferes with IL10 expression.

\section{- Discussion}

The pathophysiological mechanisms of OM, a side effect of the use of CT are not completely understood. This study aimed to clarify some aspects of the role of leukotrienes, especially cys-LTs, in the 5-FU-induced OM model, through the use of the cys-LT inhibitor MTK and the 5-lipoxygenase inhibitor (5-LOX), MK886.

In the present study, an intense inflammatory process was observed in the jugal mucosa of the animals. These observations were consistent with the peak of the inflammatory 
process described in the literature ${ }^{12,13,15,16}$. It was observed that macroscopically, when the cys-LT blocker dose was increased to $40 \mathrm{mg} /$ $\mathrm{kg} / \mathrm{d}$ for $10 \mathrm{~d}$, partial protection against OM induced by 5 -FU occurred, but not as intense as when COX-2 was blocked. This finding was not observed in the histopathological evaluation; perhaps due to the lower scores in the histopathological analysis, subtle signs of improvement were not apparent.

The immunohistochemical evaluations for iNOS2, COX-2, and IL1- $\beta$ expression in the oral mucosa showed that the expression of none of these inflammatory markers was altered by MTK or MK886 leukotriene blockade. That is, there is no influence of leukotrienes on these markers in the 5-FU induced OM model, and the inhibition of leukotrienes does not promote protection.

The involvement of leukotrienes in the regulation of iNOS gene expression has been reported in several cell types ${ }^{17-19}$. It is suggested that the action of lipoxygenase on the gene expression of iNOS has a specific relation with the cell type ${ }^{20}$. These findings may also explain why inhibition of cys-LT and 5-LOX did not reduce iNOS expression; this is probably because the cells involved in the inflammatory process of OM induced by 5-FU did not exhibit this specificity for the increase of iNOS by leukotrienes.

Studies have shown conflicting results regarding the effect of leukotriene blockade on the expression of COX-2 $2^{21-23}$. These conflicting data may be explained by the "shunt" effect between cyclooxygenase and leukotriene pathways ${ }^{9}$. Leukotriene blockade did not significantly alter the COX-2 pathway in 5-FUinduced OM.

The OM model presents increased expression of TNF- $\alpha$ and IL1- $\beta$ at its inflammatory peak (day 10$)^{13,24-28}$. The present study corroborates such results, demonstrating a significant increase in TNF- $\alpha$ and IL1- $\beta$ expression in the positive (5-FU) control group. The administration of MTK markedly reduced the expression of TNF- $\alpha$. These findings suggest that cys-LT influences the expression of TNF- $\alpha$ in the 5-FU-induced mucositis model. The presence of TNF- $\alpha$ and IL1- $\beta$ is strongly affected by COX-2 blockade, causing a large change in the expression of these proteins in OM. Blockade of 5-LOX by MK886 did not promote reduction of IL1- $\beta$ expression, but decreased the expression of TNF- $\alpha$. The participation of endogenous cys-LT in the regulation of TNF- $\alpha$ generation was demonstrated in a murine model of SCF-induced bronchial inflammation and hyperreactivity, where pre-treatment with MK886 attenuated hyperreactivity ${ }^{25}$. The diversity of cascades and the overlapping actions of various mediators ${ }^{25}$ could explain the diverse behavior of TNF- $\alpha$ and IL1- $\beta$, which exhibit similar behavior in inflammatory changes.

IL10 is a cytokine with anti-inflammatory properties in macrophages and monocytes; it decreases the production of mediators and may inhibit the presentation of antigens, although it stimulates the uptake of antigens ${ }^{26}$. It was found that the IL-10 levels in hamsters with 5-FU-induced OM were higher than those in healthy animals ${ }^{27}$. The use of COX- 2 inhibitors and cys-LTs inhibitors (at doses of 10 and $20 \mathrm{mg}$ / $\mathrm{kg} / \mathrm{d}$ for $10 \mathrm{~d}$ ) also did not significantly reduce the expression of this cytokine. However, the 5-LOX inhibitor for $4 \mathrm{~d}$, and MTK at the dose of $40 \mathrm{mg} / \mathrm{kg} / \mathrm{d}$ for $10 \mathrm{~d}$, significantly reduced IL-10 expression in relation to the 5-FU group, suggesting that a very intense blockade of cys-LTs is necessary to interfere with IL-10 expression. There is a basal level of IL-10 that is unperturbed by moderate inhibitions of cysLTs. This result suggested a partial modulatory effect of leukotrienes on IL-10. The differing IL-10 expression responses observed among 
several studies result from different pathways triggered by different stimuli ${ }^{28-30}$. In summary, IL-10 may present different responses to leukotriene stimulation according to the environment and inflammatory stimulus.

\section{Conclusions}

Inhibition of cys-LTs and 5-LOX did not play an important role in the expression of several inflammatory mediators of $\mathrm{OM}$, except for its action on TNF- $\alpha$. The findings of the present study clarify and emphasize that blockade of cys-LT by MTK does not promote protection against $\mathrm{OM}$ induced by 5-FU. Therefore, cys-LT does not play an important role in mediating 5-FU-induced OM inflammation. Blockade of 5-LOX by MK886 did not modify these findings. The effect of blocking cys-LT and 5-LOX on TNF- $\alpha$ suggests a specific modulating action on this cytokine in OM induced by $5-\mathrm{FU}$.

\section{References}

1. Scully C, Sonis, ST, Diz, PD. Oral mucositis. Oral Dis. 2006 May;12 (3):229-41. PMID: 16700732.

2. Villa A, Sonis ST. Mucositis: pathobiology and management. Curr Opin Oncol. 2015 May;27(3):159-64. PMID: 25774860.

3. Sonis, ST. New thoughts on the initiation of mucositis. Oral Dis. 2010 Oct;16:597-600. PMID: 20846150.

4. Logan, RM, Gibson, RJ, Sonis ST. Nuclear factor-kappaB (NF-kappaB) and cyclooxygenase-2 (COX-2) expression in the oral mucosa following cancer chemotherapy. Oral Oncol. 2007 Apr;43,(4):395-401. PMID: 16979925.

5. Lalla RV, Pilbeam CC,Walsh SJ, Sonis ST, Keefe DM, Peterso, DE. Role of the cyclooxygenase pathway in chemotherapy-induced oral mucositis: a pilot study. Support Care Cancer. 2010 Jan;18(1):95-103. PMID: 19404685.

6. Cianchi F, Cortesini C, Magnelli L, Fanti E, Papucci L, Schiavone N,Messerini
L.; Vannacci A, Capaccioli S, Perna F, Lulli M, Fabbroni V, Perigli G, Bechi P, Masini E. Inhibition of 5-lipoxygenase by MK886 augments the antitumor activity of celecoxib in human colon cancer cells. Mol Cancer Ther. 2006 Nov;5(11):2716-26. PMID: 17121918.

7. Jupp J, Hillier K, Elliott DH, Fine DR, Bateman $A C$, Johnson PA, Cazaly $A M$, Penrose JF, Sampson AP. Colonic expression of leukotriene-pathway enzymes in inflammatory bowel diseases. Inflamm Bowel Dis. 2007 May;13(5):537-46. PMID: 17230539.

8. Sklyarov AY, Panasyuk NB, Fomenko IS. Role of nitric oxide-synthase and cyclooxygenase/ lipooxygenase systems in development of experimental ulcerative colitis. J Physiol Pharmacol. 2011 Fev;62(1):65-73. PMID: 21451211.

9. Nakamori Y, Komatsu Y, Kotani T, Kojima, $S$, Takeuchi K. Pathogenic importance of cysteinyl leukotrienes in development of gastric lesions induced by ischemia/ reperfusion in mice. J Pharmacol Exp Ther. 2010 Apr;333(1):91-8. PMID: 20042530.

10.De Maeyer N, Kochuyt AM, Van Moerkercke W, Hiele M. Montelukast as a treatment modality for eosinophilic gastroenteritis. ActaGastroenterol Belg.2011Dec;74(4):5705. PMID: 22319970.

11.Sonis ST, Tracey C, Shklar G, Jenson J, Florine D. An animal model for mucositis induced by cancer chemotherapy. Oral Surg Oral Med Oral Pathol. 1990 Apr;69(4);37-43. PMID: 2326035.

12.Leitão RF, Ribeiro RA, Bellaguarda EA, Macedo FD, Silva LR, Oriá R B, Vale $M L$, Cunha FQ, Brito GA. Role of nitric oxide on pathogenesis of 5-fluorouracil induced experimental oral mucositis in hamster. Cancer Chemother. 2007 Apr;59(5):603-12. PMID: 16944152.

13. Medeiros CA, Leitão RF, Macedo RN, Barboza DR, Gomes A S, Nogueira NA, Alencar NM, Ribeiro RA, Brito GA. Effect of atorvastatin on 5-fluorouracilinduced experimental oral mucositis. Cancer Chemother Pharmacol. 2011 May;67(5):1085-100. PMID: 20661736.

14. Hsu SM, Raine L., Fanger $H$. Use of 
avidin-biotin-peroxidase complex (ABC) in immunoperoxidase techniques: a comparison between $A B C$ and unlabeled antibody (PAP) procedures. J Histochem Cytochem. 1981 Apr;29(4):577-80. PMID: 6166661.

15.Sonis ST. The pathobiology of mucositis. Nat Rev Cancer. 2004 Apr;4(4):277-84. PMID: 15057287.

16.Leitão RF,, Ribeiro RA, Lira AM, Silva LR, Bellaguarda EA, Macedo F D, Sousa RB, Brito GA. Glutamine and alanyl-glutamine accelerate the recovery from 5 -fluorouracilinduced experimental oral mucositis in hamster. Cancer Chemother Pharmacol. 2008;61(2):215-22. PMID: 17426972.

17.Shimpo M, Ikeda U, Maeda $Y$, Ohya K, Murakami Y, Shimada K. Effects of aspirinlike drugs on nitric oxide synthesis in rat vascular smooth muscle cells. Hypertension. 2000 May;35(5):1085-91. PMID: 10818069.

18.Chen YC, Shen SC, Chen LG, Lee TJ, Yang LL. Wogonin, baicalin, and baicalein inhibition of inducible nitric oxide synthase and cyclooxygenase-2 gene expressions induced by nitric oxide synthase inhibitors and lipopolysaccharide. Biochem Pharmacol. 2001 Jun;61(11):1417-27. PMID: 11331078.

19. Hashimoto T, Kihara M, Fujita T, Yokoyama K, Kobayashi S, Matsushita K, Tamura K, Hirawa N, Toya Y, Umemura S. Lipoxygenase products regulate nitric oxide and inducible nitric oxide synthase production in interleukin-1beta stimulated vascular smooth muscle cells. Hypertens Res. 2003 Fev;26(2):177-84. PMID: 12627879.

20.Won JS, Im YB, Khan M, Singh AK, Singh I. Involvement of phospholipase A2 and lipoxygenase in lipopolysaccharide-induced inducible nitric oxide synthase expression in glial cells. Glia. 2005 Jul;51(1):13-21. PMID: 15779087.

21.Nishikawa M, Hikasa $\mathrm{Y}$, Hori K, Tanida $\mathrm{N}$, Shimoyama T. Effect of leukotriene C4/D4 antagonist on colonic damage induced by intracolonic administration of trinitrobenzene sulfonic acid in rats. J Gastroenterol. 1995 Fev;30(1):34-40. PMID: 7719412.

22.Wallace JL, Beck PL, Morris GP. Is there a role for leukotrienes as mediators of ethanol-induced gastric mucosal damage? Am J Physiol. 1988 Jan;254(1pt1):117-23. PMID: 3337230.

23. Holma R, Salmenperä P, Riutta AV, Tanen I, Korpela R, Vapaatalo H. Acute effects of the cys-leukotriene-1 receptor antagonist, montelukast on experimental colitis in rats. Eur J Pharmacol. 2001 Oct;429:309-18. PMID: 11698051.

24.Epstein JB, Silverman SJr, Paggiarino DA, Crockett $S$, Schubert MM, Senzer NN, Lockhart PB, Gallagher MJ, Peterson DE, LevegueFG. Benzydamine $\mathrm{HCl}$ for prophylaxis of radiation-induced oral mucositis: results from a multicenter, randomized, doubleblind, placebo-controlled clinical trial. Cancer. 2001 Aug;92(4):875-85. PMID: 11550161.

25.Mellor EA, Austen KF, Boyce JA. Cysteinyl leukotrienes and uridine diphosphate induce cytokine generation by human mast cells through an interleukin 4-regulated pathway that is inhibited by leukotriene receptor antagonists. J Exp Med. 2002 Mar;195(5):583-92. PMID: 11877481.

26.Sabat R, Grüt G, Warszawska K, Kirsch S, Witte E, Wolk K Geginat J. Biology of interleukin-10. Cytokine Growth Factor Rev. 2010 Oct;21(5):331-44. PMID: 21115385.

27.De Araújo AA, Varela $H$, De Medeiros CA, De Castro Brito GA, De Lima KC, De Moura LM, De Araújo Júnior RF. Azilsartan reduced TNF- $\alpha$ and IL-1 $\beta$ levels, increased IL-10 levels and upregulated VEGF, FGF, KGF, and TGF- $\alpha$ in an oral mucositis model. PLoS One. 2015 Fev;10(2):1-16. PMID: 25689279.

28.Boyce B, Xing L. Functions of RANKL/RANK/ OPG in bone modeling and remodeling. Arch Biochem Biophys. 2008 May;473(2):139-46. PMID: 18395508.

29.Shimbori C, Shiota N, Okunishi H. Effects of montelukast, a cysteinyl-leukotriene type 1 receptor antagonist, on the pathogenesis of bleomycin-induced pulmonary fibrosis in mice. Eur J Pharmacol. 2011;650(1):424-30. PMID: 21034736.

30.Moura AP, Taddei S R, Queiroz-Junior $\mathrm{CM}$, Madeira MF, Rodrigues LF, Garlet GP, Souza DG, Machado FS, Andrade Jr I, Teixeira MM, Silva TA. The relevance of leukotrienes for bone resorption induced by 
mechanical loading. Bone. 2014;69:133-8.

PMID: 25270168.

\section{- Acknowledgement}

To Professor Ronaldo de Albuquerque

Ribeiro (in memoriam).

\section{Correspondence:}

Viviane Carvalho da Silva

Departamento de Cirurgia, Universidade Fede-

ral do Ceará

Rua Professor Costa Mendes, 1608

60430-140 Fortaleza - CE Brasil

Tel.: (55 85)99909-3136

viviancs26@gmail.com

Received: May 14, 2017

Review: July 16, 2017

Accepted: Aug 20, 2017
Conflict of interest: none

Financial source: none

${ }^{1}$ Research performed at Laboratory of Inflammation and Cancer Pharmacology, School of Medicine, Universidade Federal do Ceará (UFCE), Fortaleza-CE, Brazil. 